gescheitert, entbindet dies den Kl. nicht von der Verpflichtung, derartige Unterlagen selbst beizubringen.

Den danach grundlegenden Mangel vermag auch der in den Bilanzen der Krankenhausträger gebildete „Ausgleichsposten für Eigenmittelförderung“ nach $₫ 5$ Abs. 5 KHBV nicht zu beseitigen. Selbst wenn man - unter Zugrundelegung der Bescheinigung der R.S. GmbH Wirtschaftsprüfungsgesellschaft v. 14.10.2011 (Anlage BK 4) $\mathrm{zu}$ Gunsten des Kl. davon ausginge, dass die Bilanzen von allen seit 1966 beteiligten Trägern stets ordnungsgemäß geführt worden sind, vermag dies den Anspruch nicht hinreichend $\mathrm{zu}$ substantiieren. Denn auch die ordnungsgemäße Bilanzierung bildet insoweit nur die Bewertung eines behaupteten Eigenmittelausgleichsanspruchs durch den bilanzierenden Krankenhausträger selbst ab, trägt aber nichts zur Klärung bei, ob die tatbestandlichen Voraussetzungen des bilanzierten Anspruchs objektiv gegeben sind. Hinzu kommt im vorliegenden konkreten Einzelfall, dass der Kl. bis zuletzt nicht nachvollziehbar erklären konnte, aus welchen Gründen der bilanzierte Ausgleichsposten für Eigenmittelförderung in Höhe von zuletzt 560.568 EUR die ursprünglichen Anschaffungs- und Herstellungskosten für die fünf streitrelevanten Anlagegüter in Höhe von 419.070,33 EUR gemäß Anlage K I um mehr als 100.000 EUR übersteigt. Der insoweit in der mündlichen Verhandlung vom Kl. gegebene Hinweis, die Anlage K I bilde nur den Restbuchwert bei Übernahme der Anlagegüter durch die H. Ende 2005/Anfang 2006 ab, geht ersichtlich fehl. Die Anlage K I bildet zwar auch diese Restbuchwerte ab, beziffert daneben die ursprünglichen Anschaffungs- und Herstellungskosten aber mit 419.070,33 EUR. Die sich danach ergebende (deutliche) Differenz zum bilanzierten Ausgleichsposten für Eigenmittelförderung in Höhe von zuletzt 560.568 EUR ist klärungsbedürftig, denn abstrakt darf dieser Ausgleichsposten allenfalls die Höhe der Anschaffungs- und Herstellungskosten erreichen, soll er doch allein die (entgangene) AfA während des Zeitraums der Krankenhausförderung abbilden.

Dies zugrunde gelegt, ist der Senat auch nicht gehalten, dem nur hilfsweise für den Fall des Unterliegens gestellten und damit nicht die Bescheidungspflicht nach \86 Abs. 2 VwGO auslösenden (vgl. BVerwG, Beschl. v. 7.3.2003 - BVerwG 6 B 16.03 -, Buchholz 310 \$ 86 Abs. 2 VwGO Nr. 55) Beweisantrag stattzugeben. Der Antrag, zum Beweis der Tatsache, dass der frühere Krankenhausträger, der Landkreis J., aus Eigenmitteln Anlagegüter für das Krankenhaus vor Inkrafttreten des KHG (1972) angeschafft oder hergestellt hat, deren regelmäßige Nutzungsdauer zu diesem Zeitpunkt noch nicht abgelaufen war, eine amtliche Auskunft bei dem Landkreis J. einzuholen und diesen zur Vorlage von Unterlagen aufzufordern, stellt schon keinen zulässigen Beweisantrag dar. Er ist zwar in die schlichte Tatsachenbehauptung gekleidet, die Beschaffung der Anlagegüter sei mit Eigenmitteln des Landkreises J. erfolgt. Hierfür fehlt es aber schon, wie ausgeführt, an substantiiertem Vorbringen, das auch nur nachvollziehbare Anhaltspunkte für eine Finanzierung mit Eigenmitteln bieten könnte. Der Beweisantrag ist daher darauf gerichtet, dass im Grunde erst die Beweiserhebung selbst die entscheidungserheblichen Tatsachen und Behauptungen aufdeckt. Einem solchen bloßen Ausforschungs- bzw. Beweisermittlungsantrag muss das Gericht nicht nachgehen (vgl. BVerwG, Beschl. v. 8.3.2018 - BVerwG 9 B 25.17 -, juris, Rdnr. 31; Eyermann, VwGO, 15. Aufl. 2018, \$86, Rdnrn. 57 f.; Vierhaus, Beweisrecht im Verwaltungsprozess, 2011, Rdnrn. 55 f. m. w. N.).

Im Ergebnis muss der Klage und damit auch der Berufung gegen das klageabweisende erstinstanzliche Urt. daher der Erfolg versagt bleiben.

[...] https://doi.org/10.1007/s00350-019-5233-8

\section{Anmerkung zu Nds. OVG, Urt. v. 14.11.2018 - 13 LC 189/15 (VG Göttingen)}

\section{Kerrin Schillhorn}

Die sehr ausführliche Entscheidung des OVG Nds., die über weite Strecken andere Urteilsbegründungen zitiert, ist im Ergebnis zutreffend und berücksichtigt, dass auch kommunale Krankenhäuser als Wirtschaftsunternehmen geführt werden und insoweit auch Investitionen in Krankenhäuser tätigen bzw. getätigt haben, die im Sinne der öffentlichen Investitionsförderung übernommen werden müssten. Auch wenn der Anwendungsbereich des $₫ 8$ Abs. $2 \mathrm{Nds}$. KHG auf Ausgleich von Investitionsmitteln vor Aufnahme in den Krankenhausplan nicht sehr häufig sein dürfte, ist die Entscheidung v. 14.11.2018 doch wichtig, um die Anwendbarkeit der Regelung auf alle drei Trägergruppen zu bekräftigen. Auch inhaltlich ist der Entscheidung insoweit uneingeschränkt zuzustimmen, da überzeugende Argumente für einen Ausschluss der kommunalen Träger von dem Ausgleich für ursprünglich getätigte Investitionen nicht vorliegen. Insbesondere die von der Beklagtenseite herangezogenen Argumente, dass Investitionen durch einen kommunalen Träger auch aus öffentlichen Mitteln stammen und deshalb im Falle des Ausgleichs dieser Investitionsmittel letztlich eine Doppelfinanzierung vorliege, können jedenfalls dann nicht greifen, wenn der kommunale Krankenhausträger freie verfügbare Mittel zur Investition in die Substanz des Krankenhauses vorgenommen hat.

Ebenfalls zuzustimmen ist dem OVG bei der Beantwortung der zweiten zu entscheidenden Frage nach einer Anwendbarkeit des Ausgleichsanspruchs gem. $\$ 8$ Abs. 2 Nds. KHG auf einen insolventen Krankenhausträger. Zwar erscheint hier die vorgenommene Abgrenzung zu der bisher vorliegenden Rechtsprechung zum Ausgleichsanspruch gem. $\int 8$ Abs. 1 Nds. KHG im Falle des Ausscheidens aus dem Krankenhausplan nachvollziehbar - auch wenn die darin in Bezug genommene Rechtsprechung zu diesem Tatbestand in sich nicht ohne weiteres überzeugend ist. Insbesondere in Zeiten, in denen die Insolvenz eines Krankenhausträgers nicht zwingend bedeutet, dass damit ein Krankenhaus auch dauerhaft aus dem Krankenhausplan ausscheidet, ist es nicht überzeugend, dass allein die Insolvenz eines Krankenhausträgers bereits jegliche Ausgleichsansprüche zur Abmilderung des Ausscheidens aus dem Krankenhausplanes ausschließen soll. Zutreffend hat aber das OVG in seiner Entscheidung v. 14.11.2018 die in der bisherigen Rechtsprechung zum Ausgleichsanspruch gem. $\$ 8$ Abs. $1 \mathrm{Nds}$. KHG gefundene Argumentation nicht weiter verfolgt, sondern hier allein darauf abgestellt, dass unabhängig von der Insolvenz des Krankenhausträgers die Investitionen vor Aufnahme in den Krankenhausplan in der Tat getätigt wurden und insoweit der Ausgleichsanspruch auch im Falle der Insolvenz nicht untergehen könne. Möglicherweise kann die hier gefundene Argumentation genutzt werden, um auf den - vermutlich häufiger auftretenden - Anwendungsfall des Antrags auf Ausgleichsmittel gem. $\$ 8$ Abs. $1 \mathrm{Nds}$. KHG für den Fall des Ausscheidens des Krankenhauses aus dem Krankenhausplan nochmals neu zu bewerten. In der hier vorliegenden Konstellation ist die Entscheidung des OVG Nds. jedoch zu begrüßen. Dass dem Kl. im Ergebnis die Fördermittel nicht gewährt werden konnten, da er das Vorliegen der Tatbestandsvoraussetzungen nicht beweisen konnte, ist zwar für den Kl. unglücklich, juristisch aber ohne weiteres nachvollziehbar und nicht zu beanstanden.

Rechtsanwältin Dr. iur. Kerrin Schillhorn, MIL,

Fachanwältin für Verwaltungsrecht

und Fachanwältin für Medizinrecht,

michels.pmks Rechtsanwälte Partnerschaft mbB,

Hohenstaufenring 57, 50674 Köln, Deutschland 